\title{
Influence of measurement uncertainty propagation in current-channel-selectable multi objective optimal phasor measurement unit placement problem
}

\begin{abstract}
This paper proposes current-channel-selectable multi objective optimal Phasor Measurement Unit (PMU) placement problem with measurement uncertainty propagation. In proposed Multi Objective Optimal PMU Placement (MOOPP) problem, allocation of the current phasor channel of the PMU can be selected for reducing the total PMU placement cost. However, in practice, uncertainty of measurement makes the estimation error bigger because of use of pseudo measurement by the current channel selection. This paper proposes the optimal PMU placement method considering minimizing both the total PMU placement cost and the state estimation error with uncertainty propagation. The result of the numerical experiment demonstrates the advantage of considering the measurement uncertainty propagation, compared to the conventional method which ignores it, in IEEE New England 39-bus test system. As a result, the proposed method obtained a better Pareto solution compared to the conventional methods because of consideration of measurement uncertainty in the pseudo measurements.
\end{abstract}

Keyword: Multi objective optimization; Phasor measurement unit; State estimation 